\title{
ON THE STRUCTURE OF ALMOST NONNEGATIVELY CURVED MANIFOLDS
}

\author{
JYH-YANG WU
}

\section{Introduction}

In this paper we shall say that a compact Riemannian manifold $M$ is almost nonnegatively curved if the sectional curvature, $K_{M}$, and the diameter, $d(M)$, of $M$ satisfy

$$
K_{M} d(M)^{2}>-\varepsilon
$$

for some small positive number $\varepsilon$. In [6] and [18], Kukaya and Yamaguchi studied the fundamental groups of almost nonnegatively curved manifolds. Their results assert that there is a positive number $\varepsilon_{n}$ depending only on the dimension $n$ such that if a closed $n$-manifold satisfies $K_{M}(M)^{2}>$ $-\varepsilon_{n}$, then its fundamental group, $\pi_{1}(M)$, is almost nilpotent, i.e., $\pi_{1}(M)$ contains a nilpotent subgroup of finite index. Moreover, $M$ fibers over a $b_{1}(M)$-torus, where $b_{1}(M)$ denotes the first Betti number of $M$.

It is proved in [3] that any closed $n$-manifold $M$ with nonnegative sectional curvature is, up to a finite cover, diffeomorphic to a direct product $N \times T^{k}$, where $N$ is a simply-connected smooth $(n-k)$-manifold and $T^{k}$ is a $k$-torus. Hence, it is natural to ask whether or not this theorem still holds for almost nonnegatively curved manifolds. In general, this is not true. This can be seen from the examples: almost flat $n$-manifolds with fundamental groups of polynomial growth with degree $\geq n+1$. It is therefore clear that one needs additional assumptions to extend CheegerGromoll's theorem to the almost nonnegatively curved manifolds.

In [15], Shen and Wei considered a lower bound on the injectivity radius, $i(M)$, of $M$ and obtained the following theorem:

Theorem (Shen, Wei). Given $n, D>0$ and $i_{0}>0$, there is a positive number $\varepsilon^{*}=\varepsilon^{*}\left(n, D, i_{0}\right)$ depending only on $n, D$, and $i_{0}$ such that if $M$ is a compact Riemannian $n$-manifold satisfying

$$
d(M) \leq D, \quad i(M) \geq i_{0}, \quad K_{M}>-\varepsilon^{*},
$$

Received December 18, 1990. The author was partially supported by a National Science Foundation Grant. 
then $M$ is, up to finite cover, $C^{1}$-diffeomorphic to a direct product $N \times T^{k}$, where $N$ is a simply-connected $(n-k)$-manifold of class $C^{1}$.

Their technique is based on a recent result of Anderson and Cheeger [1] which states that the class of closed $n$-manifolds $M$ satisfying $\operatorname{Ric}_{M} \geq k$, $d(M) \leq D$, and $i(M) \geq i>0$, is $C^{\alpha}$-precompact, where $\operatorname{Ric}_{M}$ is the Ricci curvature of $M$.

In this note, we propose a weaker assumption on the lower bound of the volume, $v(M)$, of $M$. Under this new circumstance, we do not have the $C^{\alpha}$-precompactness, and Shen-Wei's technique cannot apply to this case. Here we shall employ some techniques in geometric topology about the controlled maps, manifold resolutions, and thin $h$-cobordism theorem. Our main result is

Main Theorem. Given $n, D>0$ and $v>0$, there is a positive number $\varepsilon^{*}$ such that if $M$ is a compact Riemannian $n$-manifold satisfying

$$
d(M) \leq D, \quad v(M) \geq v, \quad K_{M}>-\varepsilon^{*},
$$

then the fundamental group of $M$ is, up to a finite quotient, an almost abelian group of rank $k(1 \leq k \leq n)$, i.e., $\pi_{1}(M)$ contains a finite normal subgroup $\Phi$ such that $\pi_{1}(M) / \Phi$ is an almost abelian group of rank $k$. Moreover, if $k \neq n-3$, then a finite covering $\widehat{M}$ of $M$ is homeomorphic to the product space $N \times T^{k}$, where $N$ is a simply-connected topological $(n-k)$-manifold.

Remark 1. Recall that a group is called an almost abelian group of rank $k$ if it contains a free abelian subgroup, say $\mathbf{Z}^{k}$, of rank $k$ and finite index.

Remark 2. The first part of the Main Theorem is also obtained by Fukaya and Yamaguchi [6]. They show that $\pi_{1}(M)$ itself is almost abelian. However, our main interest is in the second part of the theorem, i.e., the structure of almost nonnegatively curved manifolds.

\section{Main tools}

For the basic notation of manifolds and Riemannian geometry, we refer to Cheeger and Ebin [2]; for the facts about Hausdorff distance, we refer to Gromov [7]; and for the definitions and basic results about resolutions, homology manifolds, and thin $h$-cobordisms, we refer to Davermann [4] and Quinn [11]-[14].

Recall that a map $f$ from a topological manifold $M$ to a space $X$ is called a resolution if it has point inverses compact, nonempty, and contractible inside any neighborhood (cf. [11]-[14]). We shall say that such a space $X$ has a resolution. 
The following results will be used in proving the Main Theorem.

Theorem 1 (Grove, Petersen, Wu [10]). Given any sequence of Reimannian n-manifolds $M_{i}$ satisfying

$$
K_{M_{i}} \geq k, \quad d\left(M_{i}\right) \leq D, \quad v\left(M_{i}\right) \geq v,
$$

there is a subsequence (still denoted by $M_{i}$ ) which converges to a metric space $(X, d)$ in the Hausdorff distance. Moreover, the sequence has the following properties:

(1) $X$ is an ENR homology n-manifold with local index 1 in the sense of Quinn (cf. [13], [14]); in particular, for $n \neq 3, X$ has a resolution.

(2) $M_{i}$ and $X$ are homotopy equivalent for large $i$.

(3) $T^{2} \times X$ is a topological $(n+2)$-manifold.

(4) Fix a metric on $T^{2}$. There are homomorphisms $f_{i}: T^{2} \times M_{i} \rightarrow$ $T^{2} \times X$ with the property

$$
\left|d(x, y)-d\left(f_{i}(x), f_{i}(y)\right)\right|<\varepsilon_{i}
$$

for all $x, y \in T^{2} \times M_{i}$ and $\lim \varepsilon_{i}=0$.

Theorem 2 (Grove, Petersen, Yamaguchi [8], [18]). Let $\left(M_{i}, g_{i}, x_{i}\right)$ be a sequence of pointed complete n-manifolds whose sectional curvature $K_{M_{i}}$ satisfies the bound $K_{M_{i}}>-\varepsilon_{i}$ with $\lim \varepsilon_{i}=0$. Let $d_{i}$ denote the metric induced by $g_{i}$. Suppose $\left(M_{i}, d_{i}, x_{i}\right)$ converges, in the pointed Hausdorff metric, to a pointed metric space $(X, d, x)$. Then $(X, d)$ is isometric to a metric product $\left(V \times \mathbf{R}^{k}, d \oplus\|\cdot\|\right)$ for some totally convex subset $(V, d)$ containing no lines, where $\|\cdots\|$ is the standard flat metric of $\mathbf{R}^{k}$.

Theorem 3 (Shen, Wei [15]). Let $\left(X_{1}, d_{1}\right)$ be a connected, locally compact, complete metric space which contains no line. Let $\mathbf{R}^{k}$ be the Euclidean space. Let $X=X_{1} \times \mathbf{R}^{k}$ with the metric $d=d_{1} \oplus\|\cdot\|$. Then any isometry $\psi$ of $(X, d)$ can be written as $\left(\psi_{1}, \psi_{2}\right)$, where $\psi_{1}$ is an isometry of $\left(X_{1}, d_{1}\right)$, and $\psi_{2}$ is an isometry of $\left(\mathbf{R}^{k},\|\cdot\|\right)$; in terms of isometry groups, $\operatorname{Isom}(X)=\operatorname{Isom}\left(X_{1}\right) \times \operatorname{Isom}\left(\mathbf{R}^{k}\right)$.

Theorem 4 (Edward, Kirby [5]). The homeomorphism group $\operatorname{Hom}(M)$ of a compact topological manifold $M$ is locally contractible.

Theorem 5 (Quinn [11]-[14]). (i) Suppose two spaces $X, Y$ have dimensions $\geq 2$. Then $X \times Y$ is a manifold if and only if $X$ and $Y$ are ENR homology manifolds of local index 1.

(ii) If $X$ is a metric space of finite dimension $\geq 4$, the following are equivalent:

(1) $X$ has a resolution. 
(2) $X \times \mathbf{R}^{k}$ is a manifold for some $k \geq 2$.

(3) $X \times \mathbf{R}^{2}$ is a manifold.

Theorem 6 (Siebenmann [11], [16]). If $f: M \rightarrow X$ is a resolution and $X$ is a manifold, then for any $\varepsilon>0, f$ can be $\varepsilon$-approximated by a homeomorphism.

Recall that an $h$-cobordism $\left(W ; \partial_{0} W, \partial_{1} W\right)$ deformation retracts to both $\partial_{0} W$ and $\partial_{1} W$. If $W \rightarrow X$ is a proper map to a metric space and $\delta>0$, then $\left(W ; \partial_{0} W, \partial_{1} W\right)$ is a $(\delta, h)$-cobordism provided that the deformations have diameter $<\delta$ in $X$. This means that the image of each arc $\{x\} \times I \subset W \times I \rightarrow W \rightarrow X$ has diameter $<\delta$ in $X$.

A map $W \rightarrow X$ is $(\delta, 1)$-connected if given a relative 2-complex $(R, S)$ and a commutative diagram

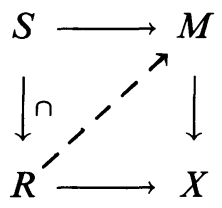

then there is a map $R \rightarrow M$ (the dotted arrow) such that the upper triangular commutes and the lower one commutes within $\delta$.

Thin $h$-cobordism theorem (Quinn [11], [12]). Suppose $X$ is a compact locally 1-connected metric space. Given any $\varepsilon>0$, there exists $a \delta=$ $\delta(X, \varepsilon)$ such that if $\left(W ; \partial_{0} W, \partial_{1} W\right) \rightarrow X$ is an $(n+1)$-dimensional, $n \geq$ $4,(\delta, 1)$-connected $(\delta, h)$-cobordism, then $W$ has a topological product structure of diameter $<\varepsilon$.

Remark 3. Let $S^{1}(r)$ be a circle with radius $r$ in $\mathbf{R}^{2}$ and $V$ a compact locally 1-connected metric space. Consider two $k$-tori $T^{k}=S^{1}(1) \times$ $S^{1}(1) \times \cdots \times S^{1}(1)$ and $\bar{T}^{k}=S^{1}\left(r_{1}\right) \times S^{1}\left(r_{2}\right) \times \cdots \times S^{1}\left(r_{k}\right)$ with $r_{i} \geq 1$, $i=1,2, \cdots, k$. Then by the definition of $(\delta, h)$-cobordism, one can see easily that $\delta\left(V \times T^{k}, \varepsilon\right) \leq \delta\left(V \times \bar{T}^{k}, \varepsilon\right)$ for all $\varepsilon>0$.

\section{Proof of the Main Theorem}

In this section we shall prove our Main Theorem by contradiction. First, we verify the almost abelian property of the fundamental groups of almost nonnegatively curved manifolds. Suppose the first part of the statement of the Main Theorem is not true. Then one can find a sequence of Riemannian $n$-manifolds $\left(M_{i}, g_{i}\right)$ satisfying

$$
K_{M_{i}}>-\varepsilon_{i}, \quad d\left(M_{i}\right) \leq D, \quad v\left(M_{i}\right) \geq v
$$


with $\lim \varepsilon_{i}=0$, such that $\pi_{1}\left(M_{i}\right)$ is not, up to a finite quotient, an almost abelian group.

Let $d_{i}$ denote the metric on $M_{i}$ induced by $g_{i}$. According to Theorem 1 , by passing to a subsequence if necessary, we can assume that $\left(M_{i}, d_{i}\right)$ converges to a compact space $(X, d)$ in the Hausdorff metric, and $\pi_{1}\left(M_{i}\right) \cong \pi_{1}(X) \cong G$ for some group $G$. To obtain a contradiction, it suffices to show that there is a finite normal subgroup $H$ of $G$ such that $G / H$ is almost abelian. Let $\widetilde{M}_{i}$ and $\widetilde{X}$ denote the universal coverings of $M_{i}$ and $X$, respectively. Endow $\widetilde{M}_{i}$ and $\widetilde{X}$ with the pullback metrics. Then the group $G$ acts isometrically on $\widetilde{M}_{i}$ and $\widetilde{X}$ as deck transformations.

Pick points $\tilde{x}_{i} \in \widetilde{M}_{i}$, and let $\pi\left(\tilde{x}_{i}\right)=x_{i}$. By taking a subsequence, we can assume that $\lim x_{i}=x \in X$. Choose a point $\tilde{x} \in \tilde{X}$ with $\pi(\tilde{x})=x$. Hence $\left(\widetilde{M}_{i}, \tilde{x}_{i}\right)$ converges to $(\tilde{X}, \tilde{x})$ in the pointed Hausdorff metric. By the Arzela-Ascoli type theorem in the appendix of [9], the action of $G$ on $\widetilde{M}_{i}$ converges to the action of $G$ on $\widetilde{X}$.

According to Theorem 2, we have $\widetilde{X}=V \times \mathbf{R}^{k}$ where $V$ is a totally convex subset of $\widetilde{X}$ and contains no lines. $\widetilde{X}$ is simply connected, and so is $V$.

Claim. $\quad V$ is compact.

Proof. If $V$ is not compact, there exists a sequence of points $p_{i}$ in $V$ such that $d\left(p_{1}, p_{i}\right)=l_{i} \rightarrow \infty$. Let $\gamma_{i}:\left[0, l_{i}\right] \rightarrow V$ be a sequence of minimal geodesics from $p_{1}$ to $p_{i}$. Set $y_{i}=\gamma_{i}\left(l_{i} / 2\right)$ and fix a point $z \in \mathbf{R}^{k}$. Note that the points $\left(p_{1}, z\right)$ and $\left(y_{i}, z\right)$ are in $\tilde{X}$.

Since $X$ is compact, one can find, for each $i$, an element $h_{i}$ in $G$ so that $d\left(h_{i}\left(y_{i}, z\right),\left(p_{1}, z\right)\right) \leq d(X)$. Let $\rho_{1}$ and $\rho_{2}$ denote the projections of $\tilde{X}=V \times \mathbf{R}^{k}$ onto the first and second factors, $V$ and $\mathbf{R}^{k}$, respectively. Set $\bar{y}_{i}=\rho_{1} h_{i}\left(y_{i}, z\right)$. Thus, $d\left(\bar{y}_{i}, p_{1}\right) \leq d(X)$. Now consider the minimal geodesics $\bar{\gamma}_{i}=\rho_{1} h_{i} \gamma_{i}$ in $V$. A subsequence of these minimal geodesics $\left(\bar{\gamma}_{i}, \bar{y}_{i}\right)$ converge to a line $(\gamma, y)$ in $V$ with $d\left(p_{1}, y\right) \leq d(X)$. Since $V$ contains no lines, this leads to a contradiction. Therefore, $V$ is compact. q.e.d.

Note that $X$ is a homology $n$-manifold, and so is $\tilde{X}$. Therefore, $V$ is a homology $(n-k)$-manifold. In particular, if $\operatorname{dim} V \leq 2$, then $V$ must be a topological manifold (cf. [4]). Hence $V$ cannot be one-dimensional, since it is a simply-connected compact manifold. If $\operatorname{dim} V \geq 4$, then $V$ has a resolution.

Now we are in a position to show that $G$ is, up to a finite quotient, almost abelian. 
The kernel of the nature projection $\phi: G=\pi_{1}(X) \subset \operatorname{Isom}(V) \times \operatorname{Isom}\left(\mathbf{R}^{k}\right)$ $\rightarrow \operatorname{Isom}\left(\mathbf{R}^{k}\right)$ is finite, since $\operatorname{Isom}(V)$ is compact. Consider the covering $\widetilde{X}=V \times \mathbf{R}^{k} \rightarrow X^{*}=\widetilde{X} / \operatorname{ker} \phi=V^{*} \times \mathbf{R}^{k}$ and the corresponding isometry group $\operatorname{Isom}\left(V^{*} \times \mathbf{R}^{k}\right)=\operatorname{Isom}\left(V^{*}\right) \times \operatorname{Isom}\left(\mathbf{R}^{k}\right)$. The projection $\phi^{*}$ : $\operatorname{Isom}\left(V^{*}\right) \times \operatorname{Isom}\left(\mathbf{R}^{k}\right) \rightarrow \operatorname{Isom}\left(\mathbf{R}^{k}\right)$ maps $G^{*}=G / \operatorname{ker} \phi \subset \operatorname{Isom}\left(V^{*} \times \mathbf{R}^{k}\right)$ isomorphically into a discrete uniform subgroup of $\operatorname{Isom}\left(\mathbf{R}^{k}\right)$. Note that $V^{*} \times \mathbf{R}^{k} \rightarrow \mathbf{R}^{k}$ induces a continuous map $X=X^{*} / G^{*} \rightarrow \mathbf{R}^{k} / \phi^{*} G^{*}$, hence $\mathbf{R}^{k} / \phi^{*} G^{*}$ is compact. Therefore, by the Bieberbach Theorem, $G^{*}$ contains a normal free abelian rank $k$ subgroup $\Gamma^{*}$ of finite index, where $\phi^{*} \Gamma^{*}$ is a lattice in the subgroup $T \cong \mathbf{R}^{k}$ of translations in $\operatorname{Isom}\left(\mathbf{R}^{k}\right)$. Thus, $G$ is, up to a finite quotient, almost abelian, and this completes the proof of the first part of the Main Theorem.

Now we continue to verify the structural part of the Main Theorem. First we summarize what we have so far by the following diagram:

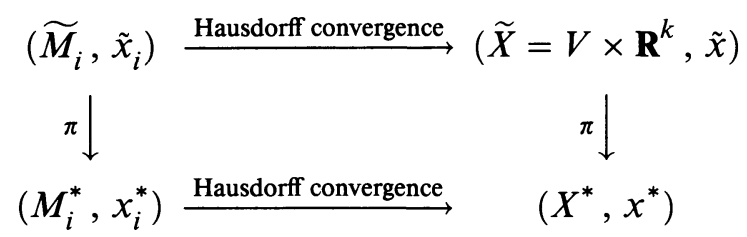

where $M_{i}^{*}=\widetilde{M}_{i} / \operatorname{ker} \phi, \pi$ denotes the covering map, and $x_{i}^{*}=\pi\left(\tilde{x}_{i}\right)$, $x^{*}=\pi(\tilde{x})$. The group $G^{*}$ acts isometrically on $M_{i}^{*}$ and $X^{*}$. Let $\mathbf{Z}^{k}=$ $\mathbf{Z} e_{1} \oplus \mathbf{Z} e_{2} \oplus \cdots \oplus \mathbf{Z} e_{k}$ be the normal free abelian subgroup $\Gamma^{*}$. Note that $\mathbf{Z}^{k}$ can be viewed as a lattice in the subgroup $T$ of translations on $\mathbf{R}^{k}=\mathbf{R} e_{1} \oplus \mathbf{R} e_{2} \oplus \cdots \oplus \mathbf{R} e_{k}$ as above. Set $\bar{X}=X^{*} / \mathbf{Z}^{k}$ and $\bar{M}_{i}=M_{i}^{*} / \mathbf{Z}^{k}$. $\bar{X}$ and $\bar{M}_{i}$ are finite coverings of $M_{i}$ and $X$, respectively, and we have the following diagram:

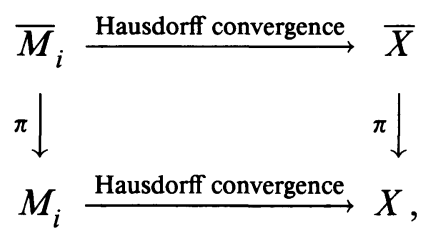

(see also [17]).

The action of $\mathrm{Z}^{k}$ on $X^{*}$ gives the fibration $V^{*} \rightarrow \bar{X} \rightarrow \widetilde{T}^{k}$, where $\widetilde{T}^{k}$ is the flat torus $\mathbf{R}^{k} / \mathbf{Z}^{k}$.

This fibration is, in general, not trivial. Namely, the action of $\mathbf{Z}^{k}$ on the first factor $V^{*}$ of $X^{*}$ is, in general, not trivial. 
Note that the isometry group $\operatorname{Isom}\left(V^{*}\right)$ may not be a Lie group in our case. Hence, the argument given in [3] cannot apply here. To overcome this difficulty, we shall investigate the fibration in a more detailed way.

Since $\mathbf{Z}^{k}$ is a lattice in the subgroup of translations on $\mathbf{R}^{k}$, we can decompose this fibration into $k$ small fibrations:

$$
\left\{\begin{array}{c}
W_{k-1} \rightarrow \bar{X} \rightarrow s_{k}^{1} \\
W_{k-2} \rightarrow W_{k-1} \rightarrow S_{k-1}^{1} \\
\ldots \ldots \ldots \ldots \cdots \\
W_{1} \rightarrow W_{2} \rightarrow S_{2}^{1} \\
V^{*} \rightarrow W_{1} \rightarrow S_{1}^{1}
\end{array}\right.
$$

where $W_{i}$ inherits a metric from $\bar{X}$, and $S_{i}^{1}$ is the circle $\mathbf{R} e_{i} / \mathbf{Z} e_{i}$, induced by the action $\mathbf{Z} e_{i}$ for $i=1,2, \cdots, k$.

Note that there is a canonical diffeomorphism $L$ from the flat torus $\widetilde{T}^{k}$ to the product space $S_{1}^{1} \times S_{2}^{1} \times \cdots \times S_{k}^{1}$ and there is a positive constant $C \geq 1$ depending only on the basis $e_{1}, e_{2}, \cdots, e_{k}$ of the lattice $\mathrm{Z}^{k}$ such that for any $x, y \in T^{k}$,

$$
\frac{1}{C} \leq \frac{d(x, y)}{d(L(x), L(y))} \leq C,
$$

i.e., $L$ is a Lipschitz map with the Lipschitz constant $C$.

Let $T^{2}$ be the 2-torus $S^{1}(1) \times S^{1}(1)$. In what follows, we shall show that there are finite coverings $\widehat{W}_{1}$ and $\widehat{S}_{1}^{1}$ of $W_{1}$ and $S_{1}^{1}$ such that $T^{2} \times \widehat{W}_{1}$ is homeomorphic to $T^{2} \times V^{*} \times \widehat{S}_{1}^{1}$ with controlled maps. Multiplying the above fibrations by the 2-torus $T^{2}$, we obtain the fibrations:

$$
\left\{\begin{array}{r}
T^{2} \times W_{k-1} \rightarrow T^{2} \times \bar{X} \rightarrow S_{k}^{1} \\
T^{2} \times W_{k-2} \rightarrow T^{2} \times W_{k-1} \rightarrow S_{k-1}^{1} \\
\\
\ldots \ldots \ldots \cdots \cdots \\
T^{2} \times W_{1} \rightarrow T^{2} \times W_{2} \rightarrow S_{2}^{1} \\
T^{2} \times V^{*} \rightarrow T^{2} \times W_{1} \rightarrow S_{1}^{1} .
\end{array}\right.
$$

Note that the spaces $T^{2} \times V^{*}, T^{2} \times X^{*}$, and $T^{2} \times W_{i}$ are closed topological manifolds. If the action of $\mathrm{Z} e_{1}$ on $V^{*}$ is trivial, then the fibration $V^{*} \rightarrow W_{1} \rightarrow S_{1}^{1}$ is trivial, and then $W_{1}$ is isometric to the metric product $V^{*} \times S_{1}^{1}$. However, this may not be the case. Hence, we need to deform the action of $\mathrm{Z} e_{1}$ to a trivial action, and then this will imply that $W_{1}$ is a, topologically, product space. 
Since the isometry group $\operatorname{Isom}\left(T^{2} \times V^{*}\right)$ is compact, there is, for any $\varepsilon_{1}>0$, an integer $m\left(\varepsilon_{1}\right)$ such that the isometry $m\left(\varepsilon_{1}\right) e_{1}$ of $T^{2} \times V^{*}$ is $\varepsilon_{1}$-close to the identity map, Id, on $T^{2} \times V^{*}$. On the other hand, $\operatorname{Isom}\left(T^{2} \times V^{*}\right) \subset \operatorname{Hom}\left(T^{2} \times V^{*}\right)$, and $\operatorname{Hom}\left(T^{2} \times V^{*}\right)$ is, by Theorem 4 , locally contractible. Hence, we can choose $\varepsilon_{1}$ so small that there is a path $\theta:[0,1] \rightarrow \operatorname{Hom}\left(T^{2} \times V^{*}\right)$ inside the $\varepsilon_{1}$-neighborhood of the identity map Id of $T^{2} \times V^{*}$ in $\operatorname{Hom}\left(T^{2} \times V^{*}\right)$ with $\theta(0)=m\left(\varepsilon_{1}\right) e_{1}$ and $\theta(1)=\mathrm{Id}$. We now consider the $m\left(\varepsilon_{1}\right)$-fold coverings $\widehat{W}_{1}$ and $\widehat{S}_{1}^{1}$ of $W_{1}$ and $S_{1}^{1}$, respectively, and can choose $m\left(\varepsilon_{1}\right)$ so large that the covering $\widehat{S}_{1}^{1}$ has length $\geq 2 \pi$, the length of $S^{1}(1)$. By using the homeomorphisms $\theta(t), t \in[0,1]$, one can deform, with small $\varepsilon_{1}$-deformation, the fibration

$$
T^{2} \times V^{*} \rightarrow T^{2} \times \widehat{W}_{1} \stackrel{p}{\longrightarrow} \widehat{S}_{1}^{1}
$$

to a trivial fibration, and the metrics on the fiber bundles $T^{2} \times \widehat{W}_{1}$ and $T^{2} \times$ $V^{*} \times \widehat{S}_{1}^{1}$ over $\widehat{S}_{1}^{1}$ are $\left(\varepsilon_{1}\right)$-close along the fibers via a homeomorphism between them, where $T^{2} \times V^{*} \times \widehat{S}_{1}^{1}$ is endowed with the product metric. Namely, there is a bundle homeomorphism $h: T^{2} \times \widehat{W}_{1} \rightarrow T^{2} \times V^{*} \times \widehat{S}_{1}^{1}$ such that if $x, y \in T^{2} \times \widehat{W}_{1}$ are in the same fiber, then

$$
|d(x, y)-d(h(x), g(y))|<\varepsilon_{1} .
$$

By $(+)$, we also have that for any $x, y \in T^{2} \times \widehat{W}_{1}$,

$$
(++) \quad|d(x, y)-d(h(x), h(y))|<\varepsilon_{1}+2 C d(p(x), p(y))
$$

provided that $d(p(x), p(y))<i\left(\widetilde{T}^{k}\right)$ the injectivity radius of $\widetilde{T}^{k}$.

Now we move to the second level:

$$
T^{2} \times W_{1} \rightarrow T^{2} \times W_{2} \rightarrow S_{2}^{1} .
$$

First, we take the $m\left(\varepsilon_{1}\right)$-fold covering $\bar{W}_{2}$ of $W_{2}$. Thus, we have the fibration

$$
T^{2} \times \widehat{W}_{1} \rightarrow T^{2} \times \bar{W}_{2} \stackrel{p}{\longrightarrow} S_{2}^{1} .
$$

Once again, we can proceed with the same argument as above. Since the isometry group $\operatorname{Isom}\left(T^{2} \times \widehat{W}_{1}\right)$ is compact, there is, for any $\varepsilon_{2}>0$, an integer $m\left(\varepsilon_{2}\right)$ such that the isometry $m\left(\varepsilon_{2}\right) e_{2}$ of $T^{2} \times \widehat{W}_{1}$ is $\varepsilon_{2}$-close to the identity isometry, Id, of $T^{2} \times \widehat{W}_{1}$. Since $\operatorname{Hom}\left(T^{2} \times \widehat{W}_{1}\right)$ is locally contractible, we can choose $\varepsilon_{2}$ so small that there is a path $\theta:[0,1] \rightarrow$ $\operatorname{Hom}\left(T^{2} \times \widehat{W}_{1}\right)$ inside the $\varepsilon_{2}$-neighborhood of the identity map Id in 
$\operatorname{Hom}\left(T^{2} \times \widehat{W}_{1}\right)$ with $\theta(0)=m\left(\varepsilon_{2}\right) e_{2}$ and $\theta(1)=$ Id. Consider the $m\left(\varepsilon_{2}\right)$ fold covering $\widehat{W}_{2}$ (resp. $\widehat{S}_{2}^{1}$ ) of $\bar{W}_{2}$ (resp. $S_{2}^{1}$ ) associated to $m\left(\varepsilon_{2}\right) e_{2}$. One can choose $m\left(\varepsilon_{2}\right)$ so large that the covering $\widehat{S}_{2}^{1}$ has length $\geq 2 \pi$. The homeomorphisms $\theta(t), t \in[0,1]$, allow us to deform with small $\varepsilon_{2}$-deformation the fibration

$$
T^{2} \times \widehat{W}_{1} \rightarrow T^{2} \times \widehat{W}_{2} \stackrel{p}{\rightarrow} \widehat{S}_{2}^{1}
$$

to a trivial fibration, the metrics on the fiber bundles $T^{2} \times \widehat{W}_{2}$ and $T^{2} \times$ $\widehat{W}_{1} \times \widehat{S}_{2}^{1}$ over $\widehat{S}_{2}^{1}$ are $\varepsilon_{2}$-close along the fibers via a homeomorphism, and the property $(++)$ still holds if one replaces $\varepsilon_{1}$ by $\varepsilon_{2}$.

Now we can proceed with the same argument on the third level, then the fourth, and so on. Finally, we shall reach the top level and obtain a trivial fibration

$$
T^{2} \times \widehat{W}_{k-1} \rightarrow T^{2} \times \widehat{X} \stackrel{p}{\longrightarrow} \widehat{S}_{k}^{1},
$$

where $\widehat{X}$ is a $\left(m\left(\varepsilon_{1}\right), m\left(\varepsilon_{2}\right), \cdots, m\left(\varepsilon_{k}\right)\right)$-fold covering of $\bar{X}$, and $\widehat{S}_{k}^{1}$, a $m\left(\varepsilon_{k}\right)$-fold covering of $S_{k}^{1}$, has length $\geq 2 \pi$. Furthermore, the metrics on the fiber bundles $T^{2} \times \widehat{X}$ and $T^{2} \times \widehat{W}_{k-1} \times \widehat{S}_{k}^{1}$ over $\widehat{s}_{k}^{1}$ are $\varepsilon_{k}$-close along the fibers via a homeomorphism. Therefore, we have

$$
\begin{aligned}
T^{2} \times \widehat{X} & \stackrel{\text { homeo }}{\approx} T^{2} \times \widehat{W}_{k-1} \times \widehat{S}_{k}^{1} \\
& \stackrel{\text { homeo }}{\approx} T^{2} \times \widehat{W}_{k-2} \times \widehat{S}_{k-1}^{1} \times \widehat{S}_{k}^{1} \\
& \cdots \cdots \cdots \cdots \\
& \stackrel{\text { homeo }}{\approx} T^{2} \times V^{*} \times \widehat{S}_{1}^{1} \times \widehat{S}_{2}^{1} \times \cdots \times \widehat{S}_{k}^{1},
\end{aligned}
$$

and the metrics on the fiber bundles $T^{2} \times \hat{X}$ and $T^{2} \times V^{*} \times \hat{S}_{1}^{1} \times \hat{S}_{2}^{1} \times$ $\cdots \times \widehat{S}_{k}^{1}$ over $\widehat{S}_{1}^{1} \times \widehat{S}_{2}^{1} \times \cdots \times \widehat{S}_{k}^{1}$ and $\varepsilon^{*}$-close along the fibers via a bundle homeomorphism, $h$, where $\varepsilon^{*}=\varepsilon^{*}\left(\varepsilon_{1}, \varepsilon_{2}, \cdots, \varepsilon_{k}\right)$ depends on the $\varepsilon_{i}$ 's such that $\varepsilon^{*} \rightarrow 0$ as all $\varepsilon_{i} \rightarrow 0$. Note that the space $\widehat{X}$ is nothing but $X^{*} / H$, where $H=\left(m\left(\varepsilon_{1}\right) \mathbf{Z} e_{1} \oplus m\left(\varepsilon_{2}\right) \mathbf{Z} e_{2} \oplus \cdots \oplus m\left(\varepsilon_{k}\right) \mathbf{Z} e_{k}\right)$. The spaces $T^{2} \times \widehat{X}$ and $T^{2} \times V^{*} \times \bar{T}^{k}$ are, in general, not Hausdorff-close, where $\bar{T}^{k}=\widehat{S}_{1}^{1} \times \widehat{S}_{2}^{1} \times \cdots \times \widehat{S}_{k}^{1}$.

Let $\widehat{M}_{i}=M_{i}^{*} / H$ and $\widehat{T}^{k}=\mathbf{R}^{k} / H$. Note that the canonical diffeomorphism $L: \widetilde{T} \rightarrow T^{k}$ can be lifted to a diffeomorphism from $\widehat{T}^{k}$ to $\bar{T}^{k}$ with the same Lipschitz constant $C$ as in (+). We have the fibration

$$
T^{2} \times V^{*} \rightarrow T^{2} \times \widehat{X} \stackrel{p}{\longrightarrow} \widehat{T}^{k} .
$$


According to Theorem 1, we also have a homeomorphism $g_{i}$ :

$$
T^{2} \times \widehat{M}_{i} \stackrel{g_{i}}{\approx} T^{2} \times \widehat{X} \stackrel{h}{\approx} T^{2} \times V^{*} \times \bar{T}^{k}
$$

such that the metrics on the fiber bundles $T^{2} \times \widehat{M}_{i}$ and $T^{2} \times V^{*} \times \bar{T}^{k}$ over $\bar{T}^{k}$ are $\left(2 \varepsilon^{*}\right)$-close along the fibers via the homeomorphism $\varphi_{i}=h \circ g_{i}$, for large $i$. Moreover, for any $x, y \in T^{2} \times \widehat{M}_{i}$,

$(+++)\left|d(x, y)-d\left(\varphi_{i}(x), \varphi_{i}(y)\right)\right|<2 \varepsilon^{*}+2 C d\left(p \circ g_{i}(x), p \circ g_{i}(y)\right)$

provided that $d\left(p \circ g_{i}(x), p \circ g_{i}(y)\right)<i\left(\widetilde{T}^{k}\right)$.

In the above argument, if the space $V^{*}$ itself is a topological manifold, then there is no need to multiply the spaces $V^{*}, \bar{X}$, and $W_{i}$ by the factor $T^{2}$, since in this case we can apply Theorem 4 directly to the homeomorphism group $\operatorname{Hom}\left(V^{*}\right)$ and go through the same argument. Therefore, if this is the case, e.g., $\operatorname{dim} V \leq 2$, then the above argument yields

$$
\widehat{M}_{i}^{\text {homeo }} V^{*} \times \bar{T}^{k}
$$

which proves the second part of the main Theorem.

From now on, we can assume that $\operatorname{dim} V=n-k \geq 4$. In this case, $V^{*}$ has a resolution $f: N \rightarrow V^{*}$ for some $(n-k)$-manifold $N$. Hence, $\pi(N) \cong \pi_{1}\left(V^{*}\right) \cong \operatorname{ker} \phi$. Since $X \times T^{2}$ is a manifold, $X^{*} \times \mathbf{R}^{2}$ is also a manifold. According to Theorem 5(ii), $X^{*}$ is, for $k \geq 2$, a topological manifold because $X^{*} \times \mathbf{R}^{2}=V^{*} \times \mathbf{R}^{k} \times \mathbf{R}^{2}$. In turn, $X$ is a topological manifold for $k \geq 2$.

Our next objective is to get rid of the factor $T^{2}$ in $(*)$ and replace the space $V^{*}$ by the manifold $N$. Once this is done, we can look at the universal covering $\widetilde{N}$ of $N$ and the $(\# \operatorname{ker} \phi)$-fold covering $\widehat{M}_{i}^{*}$ of $\widehat{M}_{i}$ associated to the subgroup $\operatorname{ker} \phi$. Note that $\tilde{N}$ is compact and $\widehat{M}_{i}^{*}=$ $\widetilde{M}_{i} / H$. Then it is easy to see that the homeomorphism from $\widehat{M}_{i}$ onto $N \times \bar{T}^{k}$ can be lifted to a homeomorphism from $\widehat{M}_{i}^{*}$ onto $\widetilde{N} \times \bar{T}^{k}$, and this will complete the proof of the Main Theorem.

Choose a positive decreasing sequence $\delta_{i}$ with $\lim \delta_{i}=0$. Since $T^{2} \times V^{*} \times \bar{T}^{k}$ is a compact manifold, by Theorem 6 , the map $\psi=$ Id $\times f \times$ Id: $T^{2} \times N \times \bar{T}^{k} \rightarrow T^{2} \times V^{*} \times \bar{T}^{k}$ can be $\delta_{i}$-approximated by a homeomorphism $\psi_{i}$. Hence, the diagram 


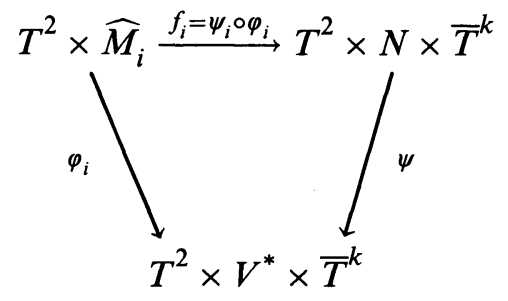

is $\delta_{i}$-commutative.

Now we can use the argument in the proof of Proposition 3.2.3 in [11] to get rid of the factor $T^{2}$ (see also the proof of Theorem 3.1 in [10]). However, it is more complicated in our case, since we do not have a fixed controlled space. This will become clear later on. Consider the following diagram:

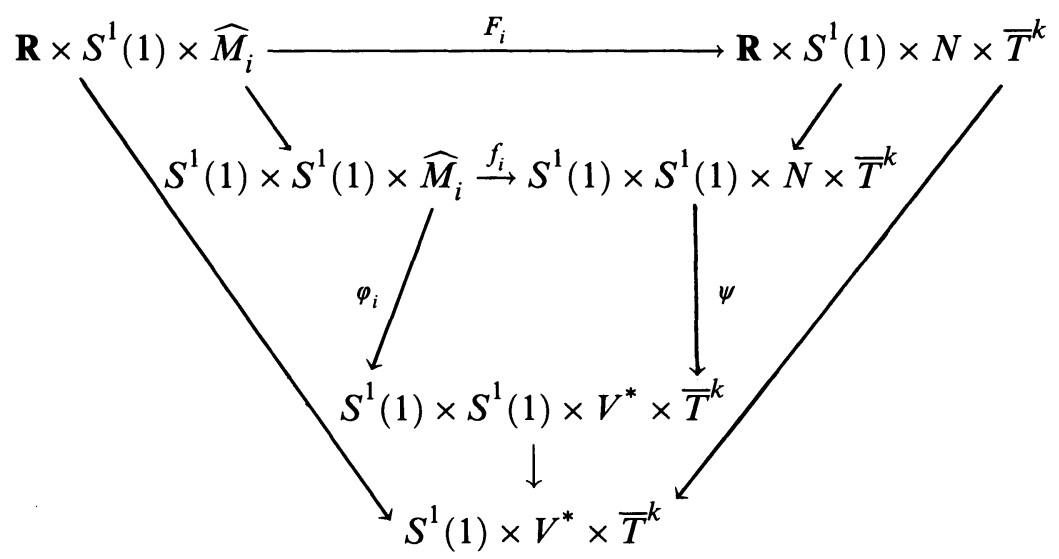

where all new maps are either lifts or projections. Choose $t_{1}$ so that $F_{i}\left(\{0\} \times S^{1}(1) \times \widehat{M}_{i}\right) \cap\left\{t_{1}\right\} \times S^{1}(1) \times N \times \bar{T}^{k}=\varnothing$, and let $W \subset(\mathbf{R} \times$ $\left.S^{1}(1) \times N \times \bar{T}^{k}\right)$ denote the region between $\partial_{0} W=F_{i}\left(\{0\} \times S^{1}(1) \times \widehat{M}_{i}\right)$ and $\partial_{1} W=\left\{t_{1}\right\} \times S^{1}(1) \times N \times \bar{T}^{k}$ in $\mathbf{R} \times S^{1}(1) \times N \times \bar{T}^{k}$. By construction, $W$ deformation retracts to both $\partial_{0} W$ and $\partial_{1} W$. By the property $(+++)$, the image of each arc $\{w\} \times I \subset W \times I \rightarrow W \stackrel{P_{i}}{\longrightarrow} S^{1}(1) \times V^{*} \times \bar{T}^{k}$ is small depending only on $\varepsilon^{*}$ and $\delta_{i}$. Thus, $W \stackrel{P_{i}}{\longrightarrow} S^{1}(1) \times V^{*} \times \bar{T}^{k}$ is a $\left(\delta_{1}^{*}, 1\right)$-connected, $\left(\delta_{1}^{*}, h\right)$-cobordism and $\delta_{1}^{*}=\delta_{1}^{*}\left(\varepsilon^{*}, \delta_{i}\right) \rightarrow 0$ as $\varepsilon^{*} \rightarrow 0$ and $\delta_{i} \rightarrow 0$ (cf. [11, Proposition 3.2.3]).

Although a different choice of the number $\varepsilon_{1}, \varepsilon_{2}, \cdots, \varepsilon_{k}$ will yield a different space $\bar{T}^{k}$, according to Remark 3 in $\S 2$, we always have $\delta\left(S^{1}(1) \times\right.$ $\left.V^{*} \times T^{k}, \varepsilon\right) \leq \delta\left(S^{1}(1) \times V^{*} \times \bar{T}^{k}, \varepsilon\right)$ for any $\varepsilon>0$, where $T^{k}$ is the 
$k$-torus $S^{1}(1) \times S^{1}(1) \times \cdots \times S^{1}(1)$ as defined in $\S 2$. Hence, we can choose $\varepsilon_{1}, \varepsilon_{2}, \cdots, \varepsilon_{k}$ so small that $\delta_{1}^{*}\left(\varepsilon^{*}, \delta_{i}\right)<\delta\left(S^{1}(1) \times V^{*} \times T^{k}, \eta\right)$ for some $\eta>0$ and for large $i \geq i(\eta)$. We shall choose the number $\eta$ latter on. The thin $h$-cobordism theorem implies that the cobordism $W$ is an $\eta$-product over $S^{1}(1) \times V^{*} \times \bar{T}^{k}$, i.e., there is a homeomorphism $\partial_{1} W \times I \rightarrow W$ which is the identity on $\partial_{1} W \times\{1\}$ and has diameter $<\eta$ in $S^{1}(1) \times V^{*} \times \bar{T}^{k}$. In particular, there is a homeomorphism $h_{i}: S^{1}(1) \times \widehat{M}_{i} \rightarrow S^{1}(1) \times N \times \bar{T}^{k}$ and an $\eta$-commutative diagram

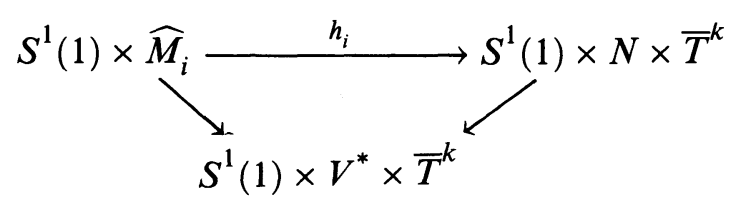

for large $i \geq i(\eta)$.

Now consider the diagram

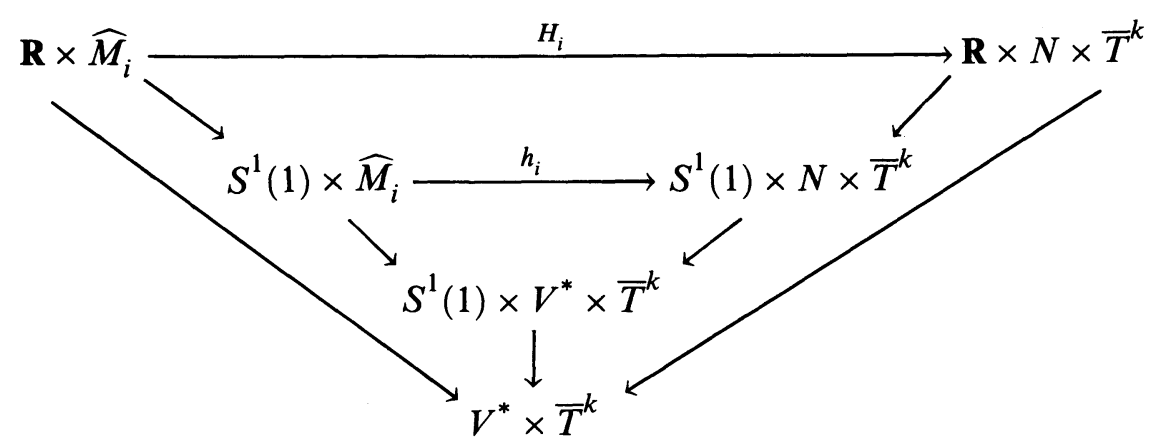

as before where all new maps are either lifts or projections. Once again, choose $t_{2}$ so that $\left.H_{i}(\{0\}) \times \widehat{M}_{i}\right) \cap\left\{t_{2}\right\} \times N \times \bar{T}^{k}=\varnothing$ and let $W \subset$ $\left(\mathbf{R} \times N \times \bar{T}^{k}\right)$ denote the region between $\partial_{0} W=H_{i}\left(\{0\} \times \widehat{M}_{i}\right)$ and $\partial_{1} W=$ $\left\{t_{2}\right\} \times N \times \bar{T}^{k}$ in $\mathbf{R} \times N \times \bar{T}^{k}$. As before, $W \stackrel{P_{i}}{\longrightarrow} V^{*} \times \bar{T}^{k}$ will be a $\left(\delta_{2}^{*}, 1\right)-$ connected $\left(\delta_{2}^{*}, h\right)$-bordism and $\delta_{2}^{*}=\delta_{2}^{*}(\eta) \rightarrow 0$ as $\eta \rightarrow 0$.

Now choose $\eta$ so small that $\delta_{2}^{*}(\eta)<\delta\left(V^{*} \times T^{k}, 1\right)$. Note that the choices above should be made in the order $\eta, \varepsilon_{1}, \varepsilon_{2}, \cdots, \varepsilon_{k}$. Although a different choice of $\varepsilon_{i}$ will change the controlled space $V^{*} \times \bar{T}^{k}$, however, Remark 3 in $\S 2$ guarantees that we can choose suitable numbers $\eta$ and $\varepsilon_{i}$ in order to apply the thin $h$-cobordism theorem as long as the length of $S_{i}^{1} \geq 2 \pi$ for $i=1,2, \cdots, k$. 
The thin $h$-cobordism theorem again implies that $W$ has product structure. In particular, $\widehat{M}_{i}$ and $N \times \bar{T}^{k}$ are homeomorphic for large $i$, and this completes the proof of the Main Theorem.

Remark. Our present proof dose not yield an estimate for $\varepsilon^{*}$ in the Main Theorem.

\section{Acknowledgment}

The author would like to thank K. Fukaya for many helpful discussions concerning this work.

\section{References}

[1] M. Anderson \& J. Cheeger, $C^{\alpha}$-compactness for manifolds with Ricci curvature and injectivity radius bounded below, J. Differential Geometry 35 (1992) 265-281.

[2] J. Cheeger \& E. Ebin, Comparison theorems in Riemannian manifolds, North-Holland Math. Library, No. 9, North-Holland, Amsterdam, 1975.

[3] J. Cheeger \& D. Gromoll, On the structure of complete manifolds of nonnegative curvature, Ann. of Math. (2) 96 (1974) 413-443.

[4] R. Davermann, Decomposition of manifolds, Academic Press, New York, 1986.

[5] R. Edwards \& R. Kirby, Deformations of spaces of embeddings, Ann. of Math. (2) 93 (1971) 63-88.

[6] K. Fukaya \& T. Yamaguchi, The fundamental groups of almost nonnegatively curved manifolds, preprint.

[7] M. Gromov, Structure metrique pour les varietes riemanniennes, Cedic/ Fernand Nathan, Paris, 1981.

[8] K. Grove \& P. Petersen V, On the excess of metric spaces and manifolds, preprint.

[9] __ Manifolds near the boundary of existence, J. Differential Geometry 33 (1991) 379-394.

[10] K. Grove, P. Petersen V \& J.-Y. Wu, Geometric finiteness theorems via controlled topology, Invent. Math. 99 (1990) 205-213.

[11] F. Quinn, Ends of maps. I, Ann. of Math. (2) 110 (1979) 275-331.

[12] _ _ Ends of maps. III, J. Differential Geometry 17 (1982) 503-521.

[13] _ Resolutions of homology manifolds, and the topological characterizations of manifolds, Invent. Math. 72 (1983) 267-284.

[14] _ An obstruction to the resolution of homology manifolds, Michigan Math. J. 34 (1987) 285-291.

[15] Z. Shen \& G. Wei, On Riemannian manifolds of almost nonnegative curvature, preprint.

[16] L. Siebenmann, Approximating cellular maps by homeomorphisms, Topology 11 (1972) 271-294.

[17] J.-Y. Wu. Convergence of Riemannian 3-manifolds under Ricci curvature bound, preprint.

[18] T. Yamaguchi, Collapsing and pinching under a lower curvature bound, Ann. of Math., to appear. 
. 